\title{
Abrechnungstipp
}

\section{Die Krux mit den Platzhalternummern}

\author{
Das GOÄ-Leistungsverzeichnis wurde 1996 zum letzten Mal aktuali- \\ siert. Mit Platzhalternummern versucht die Bundesärztekammer, \\ zumindest teilweise Aktualität in die Abrechnung zu bekommen. \\ Doch die Ziffern sind nicht bei jedem Versicherer gern gesehen.
}

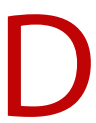
ie langen Abstände der Aktualisierungen der Gebührenordnung für Ärzte (GOÄ) erfordern eine Ergänzung fehlender neuer Leistungen. In der Regel geschieht dies durch das Bilden analoger Bewertungen gemäß Paragraf 6 Abs. 2 GOÄ. Prinzipiell kann jeder Arzt solche Analogziffern selbst generieren. Um Ärzten, Patienten wie auch Kostenträgern eine Hilfestellung bei der Analogabrechnung zu geben, hat die Bundesärztekammer (BÄK) jedoch ein "Verzeichnis der analogen Bewertungen“ veröffentlicht. Spannend daran ist, dass dort ausschließlich Leistungen, die im Einvernehmen mit dem Bundesgesundheitsministerium, dem Bundesinnenministerium und dem Verband der Privaten Krankenversicherungen e.V. gebildet wurden, aufgenommen werden. Dennoch sind die Ziffern nicht bei jedem Versicherer beliebt.

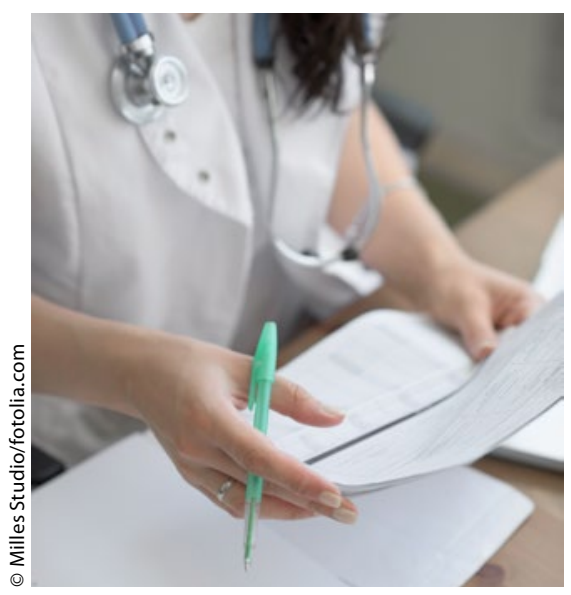

Abb. 1: Die Platzhalternummern der Bundesärztekammer machen vor allem die Suche nach analogen GOÄ-Ziffern leichter.
Das Analogverzeichnis der Bundesärztekammer enthält keine wirklichen $\mathrm{Ab}$ rechnungsnummern, sondern lediglich sogenannte „Platzhalternummern“, um die einzelne Leistung dem fachlichen Zusammenhang (Fachbereich) zuzuordnen. Da das Analogverzeichnis der BÄK bei den privaten Versicherungen bekannt ist, ist die Verwendung der "Platzhalter" bei der Rechnungserstellung möglich, aber nicht zwingend. Sie müssen also bei der Analogabrechnung nicht die Platzhalternummern verwenden. Maßgeblich ist allein die GOÄ - das Analogverzeichnis der BÄK ist nicht Bestandteil der GOÄ.

Interessant an dem Verzeichnis ist aber, dass vom Gebührenausschuss der BÄK eine Vielzahl von aktuellen Leistungen mit Sachverständigen beraten wurde und für die Aufnahme in das Verzeichnis analoger Bewertungen entsprechende Empfehlungen ausgesprochen wurden.

\section{Zusatz "analog" nicht vergessen}

Dabei erleichtern die Platzhalternummern im Praxisalltag die Suche nach der passenden Analogziffer. So kann etwa für die strukturierte Schulung eines Asthma-Patienten einfach die Platzhalternummer A 36 (strukturierte Schulung einer Einzelperson mit einer Mindestdauer von 20 Minuten bei Asthma bronchiale - einschließlich Evaluation zur Qualitätssicherung zum Erlernen und Umsetzen des Behandlungsmanagements, ... je Sitzung analog Nr. 33) aus der Liste der BÄK ausgewählt werden.

$\mathrm{Da}$ aber die GOÄ maßgeblich für die Abrechnung ist, kann die Praxis anschließend die Analogziffer - hier die GOÄ-Ziffer 33 - verwenden und dann eine eigene Analogabrechnung nach $\mathrm{Pa}-$ ragraf 6 in Verbindung mit Paragraf 12 Abs. 4 der GOÄ erstellen. Paragraf 12 Abs. 4 regelt dabei, welche Angaben die Analogabrechnung erfordert. So muss die gewählte Position entweder mit dem Zusatz „,analog“ oder „entsprechend“ gekennzeichnet werden und die erbrachte Leistung kurz, aber eindeutig beschrieben werden. Dabei muss der Leistungsinhalt entsprechend der durchgeführten Leistung geändert werden. Beispiel (Abrechnung ohne Platzhalternummer): 351 Einbringung des Kontrastmittels mittels intraarterieller Hochdruckinjektion zur selektiven Arteriografie (z.B. Nierenarterie), einschließlich Röntgenkontrolle und ggf. einschließlich fortlaufender EKG-Kontrolle, je Arterie, analog Nr. 351. Die entsprechende Platzhalternummer für diese Leistung wäre die A 353.

\section{Sonderregelung}

Für Laborleistungen ist eine Sonderregel zu beachten. Wird im Rahmen der Analogbewertung eine Leistung benutzt, die nicht in der GOÄ steht, muss die analog abgegriffene Gebührenposition durch Voranstellen des Buchstabens „A“ gekennzeichnet werden.

Da private Krankenversicherer mittlerweile einen speziellen Passus in ihre Verträge aufnehmen, können die Platzhalternummern bei der Kostenerstattung zum Problem werden. Nach besagtem Passus umfasst das Versicherungsverhältnis die Leistungen des Gebührenverzeichnisses von Nr. 1 bis Nr. 5855. Mit solchen Zusätzen in den vertraglichen Bestimmungen bzw. auch Beihilferegelungen kann die Kostenerstattung gegenüber dem Patienten für Analogabrechnungen abgelehnt werden. Patienten sollten genau in ihre Verträge schauen. Als Arzt sollten Sie in diesem Fall auf die Verwendung von Platzhalternummern verzichten und statt derer die Analogisierung entsprechend Paragraf 12 Abs. 4 GOÄ durchführen.

Dr. Dr. Peter Schlüter 